Introduction B cells classically provide humoral immunity in the form of antibody production as part of the adaptive immune response. Regulatory and antigen presenting functions of B cells have been reported before and autoantibodies are associated with autoimmune liver diseases. B cell depletion in animal models of $\mathrm{PBC}$ has highlighted the regulatory roles of $\mathrm{B}$ cells in ameliorating disease. Some evidence of efficacy of anti-B cell therapy using rituximab in human autoimmune liver diseases further supports a role for B cells. Mature B cells (Bm) subpopulations had been described in Sjogren's syndrome. However, little is known about the localisation, subsets, phenotype and function of B cells in human liver diseases.

Methods In this study we characterised the frequencies of B cell subsets in the blood and liver of patients with inflammatory and autoimmune liver diseases.

Results Frequencies of naïve mature BM1 cells were reduced in the liver compared to blood $(7.5 \% \pm 2.3$ vs. $20.2 \% \pm 2.8 \mathrm{p}=$ $0.0022)$ and $\operatorname{IgD}{ }^{\text {neg }} \mathrm{CD} 27^{\text {neg }}$ subset was increased in diseased livers compared to diseased blood $(22.9 \% \pm 6.8$ vs. $6.0 \% \pm 1.1 \mathrm{p}$ $=0.0013)$. B cells localise close to the bile ducts in PBC and reside around hepatocytes in AIH. Frequencies of regulatory B cells $\left(\mathrm{CD} 19^{\mathrm{pos}} \mathrm{CD} 24^{\mathrm{hi}} \mathrm{CD} 38^{\mathrm{hi}}\right)$ were significantly reduced in diseased blood vs. control blood $(1.8 \% \pm 0.4$ vs. $3.6 \% \pm 0.5 \mathrm{p}=$ $0.01)$ similar to recent observation in acute rheumatoid arthritis. However this population is increased in the diseased liver compared with blood $(6.2 \% \pm 0.07$ vs. $1.8 \% \pm 0.4 \mathrm{p}=0.007)$, suggesting enrichment of regulatory B cells within the inflamed liver. Liver infiltrating B cells were capable of IL-10 production. Conclusion We have characterised for the first time the heterogeneity of B cell subsets and presence of regulatory B cells and IL-10 secreting B cells in human diseased livers. We showed that $\mathrm{B}$ cells reside close to bile ducts along with other immune cells; thus B cells may play a role in biliary pathology.

Disclosure of Interest None Declared.

\section{PWE-131 FACTORS CONTRIBUTING TO VARIANCE BETWEEN ARFI ELASTOGRAPHY AND LIVER HISTOLOGY: RESULTS OF A LARGE UNSELECTED CONSECUTIVE SERIES WITH SIMULTANEOUS BIOPSY OF ARFI MEASUREMENT SITE}

${ }^{1}$ DI Sherman*, ${ }^{2}$ MJ Sangwaiya, ${ }^{3} \mathrm{P}$ Tadrous, ${ }^{2} \mathrm{P}$ Bassett, ${ }^{1} \mathrm{RD}$ Abeles, ${ }^{1} \mathrm{O}$ Kemp, ${ }^{2} \mathrm{P}$ Shorvon. ${ }^{1}$ Gastroenterology, Central Middlesex Hospital, North West London Hospitals NHS Trust, London, UK; ${ }^{2}$ Radiology, Central Middlesex Hospital, North West London Hospitals NHS Trust, London, UK; ${ }^{3}$ HIstopathology, Central Middlesex Hospital, North West London Hospitals NHS Trust, London, UK

\subsection{6/gutjnl-2014-307263.391}

Introduction ARFI ${ }^{\mathrm{TM}}$ (Acoustic Radiation Force Impulse) elastography is a widely applicable technique for the non-invasive assessment of liver fibrosis, which has been well validated in viral hepatitis patients. As with transient elastography, the predictive value of this technique falls in intermediate stages of fibrosis, and shear velocity readings may also be affected by a number of other factors. However, few studies have systematically exam: ined the causes of the observed variance with liver histology. We report the results of a large unselected series, in which liver stiffness and histology have been sampled simultaneously from the same region of liver tissue.

Methods One hundred and eighty six unselected, consecutive secondary care referrals underwent simultaneous elastography and liver biopsy from the same right lobe liver window, both performed or supervised by a single senior radiologist in all cases. ARFI shear velocity measurements were made using a standard 10 observation technique, and biopsies taken using an $18 \mathrm{G}$ Biopince $^{\mathrm{TM}}$ needle. All biopsies were reviewed by a single specialist histopathologist. Clinical, laboratory, elastographic and histological data were analysed retrospectively. ARFI/histological variance (AHV) was defined as a difference of more than 1 Metavir or 2 Ishak stages from that predicted by ARFI, according to standard calibration. ${ }^{1}$

Results Aetiologies were 99 viral hepatitis, 39 autoimmune (AILD) and other in 48. AHV was seen in 56(30.1\%), of which $46(82 \%)$ showed a lower histological stage than predicted. AHV was not associated with age, gender, or ARFI measurement depths. Inflammation (ALT, necroinflammation), steatosis (US echogenicity, histology), suboptimal ARFI quality (IQR/median $>0.3$ ) and AILD aetiology were significantly more common in AHV ( $\mathrm{p}=0.01,0.007,<0.001$ and 0.018 , respectively). Two or more of these variables were present in $61 \%$ of variants, compared with $26.9 \%$ of non-variants ( $p<0.001$ ).

Conclusion These simultaneous paired data show that ARFI/ histological variance is common and influenced by aetiology, inflammation, steatosis and technical quality. It is more common in active autoimmune liver disease than in viral hepatitis. Assuming that histology is a true "Gold standard", taking these 4 factors into account when interpreting ARFI scores will assist in assessing the predictive reliability of elastography, and hence in clinical decision making with regard to liver biopsy and treatment. Further prospective studies with paired ARFI/histology sampling are warranted to confirm these findings.

\section{REFERENCE}

1 Friedrich-Rust M, et al. Radiology 2009:252: 595-604

Disclosure of Interest None Declared.

\section{PWE-132 ASSOCIATION BETWEEN SMOKING AND LIVER FIBROSIS IN PRIMARY BILIARY CIRRHOSIS}

${ }^{1} \mathrm{~A}$ Mantaka, ${ }^{1} \mathrm{M}$ Koulentaki, ${ }^{1} \mathrm{D}$ Sifaki-Pistolla, ${ }^{1} \mathrm{~A}$ Voumvouraki, ${ }^{1} \mathrm{E}$ Marinidou, ${ }^{1} \mathrm{~N}$ Papiamonis, ${ }^{2} \mathrm{M}$ Tzardi, ${ }^{1} \mathrm{E}$ Kouroumalis*. ${ }^{1}$ Gastroenterology, University of Crete Medical School, Heraklion, Greece; 'Pathology, University of Crete Medical School, Heraklion, Greece

\subsection{6/gutjnl-2014-307263.392}

Introduction Conflicting data for the role that cigarette smoking may play in Primary Biliary Cirrhosis (PBC) have been reported. Some studies have suggested an association of smoking with a more advanced fibrotic stage. The aim of the present study therefore was to assess the association between smoking and a) the severity of histological findings at the time of diagnosis, b) the immunological features of a genetically homogeneous and geographically defined population of PBC patients.

Methods Smoking history data were collected from 171 PBC patients of Cretan origin (163 female) using a standardised questionnaire. Diagnosis was based on standard biochemical, Immunological and histological criteria. Liver biopsy was performed in 148 patients at diagnosis. Liver fibrosis and histological inflammatory activity were semi-quantified according to a METAVIRbased classification system. Odds ratios (OR) were assessed using logistic regression analysis.

Results Smoking history prior to diagnosis was reported in 56 patients $(32,7 \% \%)$. Twenty-six patients $(15,2 \%)$ were active smokers at diagnosis. Male gender (AOR 8.19, 95\% CI: 3.01411.937), alcohol intake $>20 \mathrm{~g} / \mathrm{d}$ (AOR, 2.20, 95\% CI: 1.029 4.099), severe steatosis (AOR, 5.31, 95\% CI: 2.019-9.919)), and F3-F4 fibrosis stage (AOR 1.21 95\% CI: 1.015-3.031), but 
not piecemeal necrosis grade, bile duct paucity and cholangitis, or immunological laboratory data, were associated with smoking history. Multiple logistic regression analysis identified smoking intensity, years of passive smoking and significant necroinflammatory histological activity as independent risk factors of advanced liver fibrosis (F3-F4 stage) at diagnosis, adjusted for age, gender, BMI and alcohol consumption. For every pack-year increase in smoking intensity there was a 3.2 times higher likelihood of advanced fibrosis (95\% CI: 2.018-6.294).

Conclusion Our study results confirm the previously reported link between smoking history and the risk of advanced liver fibrosis at diagnosis in PBC. The mechanism by which smoking may accelerate the histological progression of $\mathrm{PBC}$ is unknown and larger studies are needed to define it.

Disclosure of Interest None Declared.

\section{PWE-133 HEPATOCELLULAR CARCINOMA: IMPACT OF NICE GUIDELINES ON ASSESSING AND OPTIMISING SURVEILLANCE}

E Johnston*, R Khan, J Ingoe, G Tritto. Gastroenterology, Guy's and St Thomas' NHS Foundation Trust, London, UK

\subsection{6/gutjnl-2014-307263.393}

Introduction Screening for hepatocellular carcinoma (HCC) is only deemed cost-effective in patients at high risk. However, the criteria for identifying patients at risk, particularly in relation to non-cirrhotic hepatitis B, remains ill defined and different international guidelines are not consistent. Recent NICE guidelines proposed offering surveillance to patients with significant fibrosis $(\geq \mathrm{F} 2)$ or age $>40$ with either high viral load or family history of HCC. The aim of this study was to evaluate how the new NICE guidelines can impact on current practice and on the effectiveness of our surveillance program.

Methods All patients who underwent an abdominal ultrasound for HCC surveillance from $1^{\text {st }}$ September 2012-31 $1^{\text {st }}$ August 2013 were identified and the indications evaluated. In a subgroup of these patients with hepatitis B the appropriateness of screening was assessed according to the 2013 NICE guidelines. From the same period, all HCC diagnosed were identified and cross matched with our surveillance list.

Results In the period under study, 1280 surveillance ultrasounds (606 in cirrhosis, 628 in non cirrhotic hepatitis B or C, 46 other) led to 5 diagnoses of HCC. All HCC were found in cirrhotic patients (annual incidence 1.65\%). Seventeen further HCC were diagnosed from emergency admissions or referred from elsewhere. One patient had non cirrhotic hepatitis B (78yr old male with a long history of hepatitis $B$ ) whilst all the remaining were cirrhotic (2 with hepatitis B). In a randomly selected sample of 200 patients undergoing surveillance ultrasounds, $62 \%(n=124)$ had hepatitis B and 76 had cirrhosis of other aetiology. Of the patients with hepatitis $B$, an assessment of specific risk factors, particularly level of fibrosis, was not documented in 50 (40\%). A further 48 (39\%) patients were retrospectively deemed low risk according to NICE guidance.

Conclusion The number of HCC diagnosed through surveillance was low, but still cost effective in cirrhotic patients. The main risk factor for HCC in this cohort was cirrhosis (96.5\%). In fact, only 1 non cirrhotic patient with hepatitis B developed HCC and this was on a background of likely advanced fibrosis. None of the HCC from surveillance would have been missed if NICE criteria were used. It is important that patients with chronic hepatitis $\mathrm{B}$ all undergo assessment of fibrosis, along with clear documentation of other known risk factors in order to enable better risk stratification. In our experience following NICE guidance to identify high risk patients for surveillance will pick up all cases of HCC and decrease cost by reducing the number of unnecessary ultrasound scans being carried out.

\section{REFERENCE}

NICE clinical guideline 165 - Hepatitis B (chronic) June 2013 (http://www.nice.org.uk/ nicemedia/live/14191/64234/64234.pdf)

Disclosure of Interest None Declared.

\section{PWE-134 OVERVIEW OF EFFICACY AND COST EFFECTIVENESS OF NURSE LED DAY CASE ABDOMINAL PARACENTESIS SERVICE AT GLOUCESTERSHIRE HOSPITAL NHS FOUNDATION TRUST}

F Tahir*, C Hollywood, D Durrant. Gastroenterology Department, Gloucestershire NHS Foundation Trust, Gloucester, UK

\subsection{6/gutjnl-2014-307263.394}

Introduction Liver disease is the fifth most common cause of death in England and Wales ${ }^{1}$ and is one of the important causes of acute hospital admissions. Ascites is a common complication of Cirrhosis and patient with refractory ascites often require therapeutic paracentesis for symptomatic relief. In majority of UK hospitals, therapeutic paracentesis is done via planned admission to an acute medical bed which often causes delay waiting for a bed. In one study, the average hospital stay for therapeutic paracentesis was 5.5 days, ${ }^{2}$ as they have to wait for abdominal ultrasound to mark a suitable drain site. This not only leads to patient dissatisfaction but also has significant cost implications for the Trust.

Methods Retrospect patient's data from 2010-2013 was reviewed who attended our Ambulatory Day Unit for therapeutic paracentesis.

Results The service was set up in 2010 at the Gloucestershire Royal Hospitals with a view to perform 1-2 paracentesis weekly, which then gradually expanded to up to12-14 paracentesis week. Paracentesis was performed by an experienced and dedicated liver specialist nurse, using aseptic technique and under Human Albumin solution (100 mls of 20\%) cover for every 3 litres of ascites drained. 111 patients have so far benefited from the service and 764 procedures are performed with 6 failed attempts in a single patient only with malignant ascites. There have been no complications. $63(56.7 \%)$ patients required paracentesis once every two or more months whilst 19 (17.1\%) needed weekly drains. The most number of attendances by a single patient was 62 . The day case service is cost effective and has saved the Trust over $£ 30,000$ per annum. Patients love this service as it is easily accessible and has significantly reduced the waiting time for paracentesis as the service is available to patients within a few days and they are able to self-refer.

Conclusion We found the nurse led day case therapeutic paracentesis service is safe and cost effective. It is easily accessible and is liked by the patients as it gives them more control on their treatment and better continuity of their care. It has also a potential to provide training opportunities to junior doctors. We advocate setting up similar services in other NHS Trusts

\section{REFERENCES}

1 The Health Survey for England 2007

2 Hill $S$, et al. Developing a nurse led, day-case, abdominal paracentesis service. Cancer Nursing Practice June 2013;12(5)

Disclosure of Interest None Declared. 